\title{
Leucine aminopeptidase of the human blood flukes, Schistosoma mansoni and Schistosoma japonicum ${ }^{\text {is }}$
}

\author{
Elaine McCarthy ${ }^{\mathrm{a}, \mathrm{b}}$, Colin Stack $^{\mathrm{a}, \mathrm{b}}$, Sheila M. Donnelly ${ }^{\mathrm{a}}$, Sean Doyle ${ }^{\mathrm{b}}$, Victoria H. Mann ${ }^{\mathrm{c}}$, Paul \\ J. Brindley ${ }^{c}$, Michael Stewart ${ }^{\mathrm{d}}$, Tim A. Day ${ }^{\mathrm{e}}$, Aaron G. Maule ${ }^{\mathrm{d}}$, John P. Dalton ${ }^{\mathrm{a}, \mathrm{f}, *}$ \\ ${ }^{a}$ Molecular Parasitology Unit, School of Biotechnology, Dublin City University, Dublin 9, Ireland \\ ${ }^{\mathrm{b}}$ Department of Biology, National Institute for Cellular Biotechnology, National University of Ireland, Maynooth, Co. Kildare, Ireland \\ ${ }^{\mathrm{c}}$ Department of Tropical Medicine, Tulane University Health Sciences Center, New Orleans, LA 70112, USA \\ ${ }^{\mathrm{d}}$ School of Biology and Biochemistry, Medical Biology Center, The Queen's University of Belfast, 97 Lisburn Road,Belfast BT9 7BL, UK \\ ${ }^{\mathrm{e}}$ Department of Biomedical Sciences, Iowa State University,Ames, IA 50011, USA \\ ${ }_{\mathrm{f}}^{\mathrm{f}}$ Institute for the Biotechnology of Infectious Diseases (IBID), University of Technology Sydney, Westbourne Street, Gore Hill, Sydney, Australia
}

Received 24 October 2003; received in revised form 28 January 2004; accepted 29 January 2004

\begin{abstract}
An array of schistosome endoproteases involved in the digestion of host hemoglobin to absorbable peptides has been described, but the exoprotease responsible for catabolising these peptides to amino acids has yet to be identified. By searching the public databases we found that Schistosoma mansoni and Schistosoma japonicum express a gene encoding a member of the M17 family of leucine aminopeptidases (LAPs). A functional recombinant $S$. mansoni LAP produced in insect cells shared biochemical properties, including pH optimum for activity, substrate specificity and reliance on metal cations for activity, with the major aminopeptidase activity in soluble extracts of adult worms. The $\mathrm{pH}$ range in which the enzyme functions and the lack of a signal peptide indicate that the enzyme functions intracellularly. Immunolocalisation studies showed that the S. mansoni LAP is synthesised in the gastrodermal cells surrounding the gut lumen. Accordingly, we propose that peptides generated in the lumen of the schistosome gut are absorbed into the gastrodermal cells and are cleaved by LAP to free amino acids before being distributed to the internal tissues of the parasite. Since LAP was also localised to the surface tegument it may play an additional role in surface membrane re-modelling.
\end{abstract}

(C) 2004 Australian Society for Parasitology Inc. Published by Elsevier Ltd. All rights reserved.

Keywords: Aminopeptidase; Proteases; Haemoglobin digestion; Parasites; Helminths; Trematodes; Schistosomes

\section{Introduction}

Schistosomiasis is a chronic parasitic disease widespread in tropical and sub-tropical regions such as Africa, the Middle East, South America and South East Asia. In 1999, the World Health Organisation estimated that 652 million people were at risk, with 193 million actually infected and about 20 million clinically ill from schistosomiasis (WHO, 1999). The disease is caused by blood flukes of the genus Schistosoma, of which five species, Schistosoma mansoni,

\footnotetext{
The Genbank accession number for the Schistoma Mansoni expression construct described in this manuscript is AY523600.

* Corresponding author. Address: Institute for the Biotechnology of Infectious Diseases (IBID), University of Technology Sydney, Westbourne Street, Gore Hill, Sydney, Australia. Tel.: +61-2-9514-4142; fax: +61-29514-4201.

E-mail address: john.dalton@uts.edu.au (J.P. Dalton).
}

Schistosoma japonicum, Schistosoma haematobium, Schistosoma intercalatum and Schistosoma mekongi, are of medical importance to humans (Engels et al., 2002; Ross et al., 2002). Our laboratories have been particularly interested in proteases that participate in proteolysis of host hemoglobin, an essential source of nutrient for the schistosome parasite. Maturing and adult schistosomes live in the blood vessels of their human hosts where they feed on red blood cells. The ingested red blood cells are lysed by hemolysins within the oesophagus of the parasites and hemoglobin that is released flows into caeca of the schistosome (Dalton et al., 2004). Several proteolytic enzymes participate in the progressive catabolism of hemoglobin to amino acids that are subsequently utilised in parasite metabolic processes. A model for this catabolic process proposes that the initial cleavages of hemoglobin 
are performed by endopeptidases, including cathepsins B, L and $\mathrm{D}$, liberating short peptides that are subsequently hydrolysed by exopeptidases into absorbable dipeptides and amino acids (Brindley et al., 1997; Tort et al., 1999; Sajid and McKerrow, 2002). Whereas we and others have characterised the exopeptidase dipeptidyl peptidase I, (= cathepsin C), which is secreted from the parasite gastrodermis into the gut and most likely is the enzyme that cleaves dipeptides from fragments of host hemoglobin (Hola-Jamriska et al., 1998, 1999, 2000), little is known of the putative aminopeptidase that is crucial to the final stage of liberating free amino acids.

Aminopeptidase activity has been demonstrated in soluble extracts of various stages of the $S$. mansoni life cycle (Auriault et al., 1982; Damonneville et al., 1982; Xu and Dresden, 1986; Xu et al., 1988, 1990). Since the enzyme displayed enhanced activity against synthetic substrates containing an N-terminal leucine it has been termed leucine aminopeptidase (LAP) (Auriault et al., 1982). The LAP activity detected in schistosomula and adult extracts was shown to be immunogenic during infections since the enzyme was reactive with antibodies in the serum of infected rats and humans (Auriault et al., 1982; Damonneville et al., 1982; Doenhoff et al., 1988). LAP activity is also expressed by schistosome eggs and has been implicated in the emergence of the schistosome miracidium since hatching can be blocked by bestatin, a general inhibitor of aminopeptidases (Xu and Dresden, 1986; Xu et al., 1988, 1990).

In this study, we isolated a cDNA encoding an S. mansoni aminopeptidase that is a member of the metalloprotease M17 family of LAPs (Strater and Lipscomb, 1998). Our characterisation of the recombinant and native LAP shows that it is predominantly expressed in the gastrodermis of the adult worms, and indicates that we have identified the schistosome enzyme responsible for the final stage in the catabolism of host hemoglobin. We discuss these findings in the context of our model of hemoglobin proteolysis in these blood-feeding parasites, and of previous reports of aminopeptidase activity in schistosomes.

\section{Materials and methods}

\subsection{Parasite extracts, protein preparations and infection sera}

Soluble extracts of $S$. mansoni cercariae and adult worms and $S$. japonicum adult worms were prepared in phosphate buffered saline (PBS, pH 7.3) after three freeze thaw cycles and sonication cycles on ice as described previously (Dalton et al., 1996). Insect cell-expressed S. mansoni asparaginyl endopeptidase (Sm32) (Brindley et al., 1997; Tort et al., 1999) was purified in our laboratory (Stack, Dalton and Doyle, unpublished). Pig kidney LAP was obtained from Sigma Chemical Co (Dorset, Poole).

\subsection{Fluorogenic substrate assay and fluorographic gel analysis for the detection of aminopeptidase activity}

Aminopeptidase activity of $S$. mansoni or S. japonicum soluble extracts, porcine kidney LAP or recombinant $S$. mansoni LAP was measured using the fluorogenic peptidyl substrates L-leucine (Leu)-7-amido-4-methylcoumarin hydroxide (NHMec), L-alanyl (alanine)-NHMec, L-tyrosyl (tyrosine)-NHMec and L-prolyl (proline)-NHMec (Sigma). The assays were carried out by incubating samples in a reaction mix of $10 \mu \mathrm{M}$ fluorogenic peptidyl substrate, $0.5 \mathrm{mM} \mathrm{MgCl}_{2}$ and $0.1 \mathrm{M}$ Tris $-\mathrm{HCl}(\mathrm{pH}$ 8.5) in a final volume of $1 \mathrm{ml}$ at $37^{\circ} \mathrm{C}$ for $30 \mathrm{~min}$ as described before (Gavigan et al., 2001). A standard curve was prepared using $0-10 \mu \mathrm{M}$ NHMec and enzyme units presented nmol $\mathrm{NHMec}$ released $/ \mathrm{min} / \mathrm{ml}$.

For the activation/inhibition studies, the enzyme was incubated with the divalent cations $\mathrm{MgCl}_{2}, \mathrm{MnCl}_{2}$ and $\mathrm{ZnCl}_{2}$, the metal chelating reagents 1,10-phenanthroline, and $N, N$-ethylenediaminetetraacetic acid (EDTA) or the specific aminopeptidase inhibitor bestatin at $37^{\circ} \mathrm{C}$ for 10 min prior to addition of the fluorogenic peptidyl substrate to the assay mix and incubation for $30 \mathrm{~min}$. The $\mathrm{pH}$ profile was determined by incubating enzyme in $0.1 \mathrm{M}$ Tris $-\mathrm{HCl}$ buffers ranging from $\mathrm{pH} 6.75$ to 9.4 .

To characterise aminopeptidase activity in gels soluble extracts of adult $S$. mansoni and S. japonicum, recombinant S. mansoni LAP and pig kidney LAP were separated on $10 \%$ native polyacrylamide gels, which were then washed in $0.1 \mathrm{M}$ Tris $-\mathrm{HCl}\left(\mathrm{pH} 8.5\right.$ ) containing $0.5 \mathrm{mM} \mathrm{MgCl}_{2}$ and incubated for $10 \mathrm{~min}$ in the same buffer containing $50 \mu \mathrm{M}$ L-leucine-NHMec as described (Curley et al., 1994; Acosta et al., 1998). Fluorescent bands representing enzymes with LAP activity were visualised under UV light and photographed.

\subsection{Identification and analysis of the cDNA encoding schistosome leucine aminopeptidase}

A cDNA encoding an $S$. mansoni aminopeptidase (GenBank U83906) was discovered by searching the public databases with the peptide sequence NTDAEGRL that represents the conserved active site region of aminopeptidases (Kim and Lipscomb, 1993; Strater and Lipscomb, 1998). Analysis of the $S$. mansoni aminopeptidase sequence was performed using sequence retrieval system (SRS), Blast tools, and MEROPS at http://merops.sanger.ac.uk. The Bos taurus LAP (bovine lens LAP, BILAP) sequence and structural data were employed to locate catalytic domains and critical active site residues (Kim and Lipscomb, 1993; Taylor, 1993). The phylogenetic relationship between selected LAPs of the M17 family of aminopeptidases was examined using selected C-terminal domains only. Sequence similarities within the catalytic domains were determined using the BLAST algorithm and the selected sequences were then aligned using the ClustalX 1.81 
program (Thompson et al., 1997). The PHYLIP tree was calculated using the bootstrapping method, with the number of bootstrapping trials set at 1000 as recommended. The program also corrected for multiple substitutions.

\subsection{Construction of cDNA expression vector}

Primers were designed to amplify the full-length cDNA encoding $S$. mansoni LAP using the DNA sequence information deposited in GenBank (accession no. U83906) as follows: Forward (5'-GCGGCCTCGAGATGAGC GTTGTCACTCCCGTGTTCCCG-3') and Reverse (5'GCCGCTCTAGATTAGTGGTGGTGGTGGTGGTGGG GCCCCTTGAAACTTAATCG- $3^{\prime}$ ). The forward and reverse primers incorporated the restriction sites Xho I and $X b a$ I (underlined), respectively. The forward primer incorporated a start codon (bold) which was not present in the published sequence (see Section 3) and the reverse primer contained a sequence encoding a $\mathrm{His}_{6}$ tag (double underlined) and a stop codon (also bold). Amplification of the LAP cDNA from an $S$. mansoni cDNA library was performed under the following conditions: initial denaturation $\left(94^{\circ} \mathrm{C}, 5 \mathrm{~min}\right)$, followed by 30 cycles of denaturation $\left(94{ }^{\circ} \mathrm{C}, 1.5 \mathrm{~min}\right)$, annealing $\left(55^{\circ} \mathrm{C}, 1.5 \mathrm{~min}\right)$ and primer extension $\left(72{ }^{\circ} \mathrm{C}, 1.5 \mathrm{~min}\right)$, followed by a final extension step $\left(72{ }^{\circ} \mathrm{C}, 7 \mathrm{~min}\right)$. PCR products were cloned into pGem-T vector (Promega, USA), and the LAP gene subsequently excised from the plasmid using restriction endonucleases $X h o I$ and $X b a I$ and cloned into the baculovirus expression transfer vector pBlueBac4.5 (Invitrogen). The insert of the pBlueBac4.5 construct was sequenced to confirm orientation and identity of the LAP cDNA.

\subsection{Production and purification of recombinant S. mansoni LAP in baculovirus expression vector system}

Spodoptera frugiperda $\left(\mathrm{Sf}_{9}\right)$ insect cells (Invitrogen) were transformed with pBlueBac4.5 plasmid construct incorporating the $S$. mansoni LAP cDNA as previously described (Ennis et al., 2001). Cell suspensions were lysed in the presence of $0.05 \%(\mathrm{v} / \mathrm{v})$ Tween $^{\circledR} 20$ (Sigma) detergent in $50 \mathrm{mM} \mathrm{NaH}{ }_{2} \mathrm{PO}_{4}, 300 \mathrm{mM} \mathrm{NaCl}$, and $10 \mathrm{mM}$ imidazole ( $\mathrm{pH} 8.0$, lysis buffer), and then incubated on ice for $30 \mathrm{~min}$. The cell lysate was centrifuged at $14,000 \times g$ for $30 \mathrm{~min}$ and the supernatants passed over a $1 \mathrm{ml} \mathrm{Ni-NTA}$ resin column, and the column washed with five volumes of $50 \mathrm{mM} \mathrm{NaH}{ }_{2} \mathrm{PO}_{4}, 300 \mathrm{mM} \mathrm{NaCl}$, and $20 \mathrm{mM}$ imidazole (pH 8.0). Bound protein was eluted using $250 \mathrm{mM}$ imidazole in $50 \mathrm{mM} \mathrm{NaH} \mathrm{PO}_{4}$ and $300 \mathrm{mM} \mathrm{NaCl}(\mathrm{pH}$ 8.0). The production and purification of recombinant S. mansoni LAP was monitored by Coomassie Blue-stained SDS-PAGE (Dalton et al., 1996). Protein concentrations were determined using a bicinchoninic protein assay kit (Pierce, Rockford, IL) with bovine serum albumin $\left(0-2 \mathrm{mg} \mathrm{ml}^{-1}\right)$ as protein standard.

\subsection{Preparation of polyclonal antiserum to S. mansoni LAP (anti-SmLAP)}

Polyclonal antiserum to $S$. mansoni LAP was obtained by immunising a rabbit four times at 3-week intervals, subcutaneously, with purified recombinant protein $(50 \mu \mathrm{g})$ formulated in Freunds Complete or Incomplete adjuvant. Serum was obtained 10 days after the final immunisation.

\subsection{Immunoblotting}

Soluble $S$. mansoni and $S$. japonicum extracts $(10 \mu \mathrm{g})$ and purified recombinant LAP $(2.0 \mu \mathrm{g})$ were resolved on $12 \%$ reducing SDS-PAGE gels and then transferred to nitrocellulose membrane. The membranes were blocked in $5 \%(\mathrm{w} / \mathrm{v})$ milk powder/PBS plus $0.05 \%$ (v/v) Tween ${ }^{\circledR} 20$ (PBST) for $1 \mathrm{~h}$ and then probed with rabbit anti-S. mansoni LAP serum (1:6000) or pre-immunisation serum (control) for $1 \mathrm{~h}$ at room temperature. Bound antibody was visualised using peroxidase-conjugated goat anti-rabbit IgG (1:1000) and diaminobenzidine $/ \mathrm{H}_{2} \mathrm{O}_{2}$ (Sigma, Dorset, UK) as the chromogenic substrate (Brady et al., 1999a,b).

\subsection{Confocal scanning laser microscopy}

Adult $S$. mansoni were flat-fixed in $4 \%$ paraformaldehyde (PFA) in PBS (pH 7.2) for $1 \mathrm{~h}$ and then free-fixed for a further $3 \mathrm{~h}$ at $4{ }^{\circ} \mathrm{C}$. They were washed in antibody diluent (AbD: PBS with $0.1 \%$ bovine serum albumin, $0.3 \%$ Triton $\mathrm{X}-100$ and $0.1 \%$ sodium azide) for $24 \mathrm{~h}$ before being incubated for $72 \mathrm{~h}$ at $4{ }^{\circ} \mathrm{C}$ in anti-SmLAP serum (diluted 1:500) and subsequently washed in $\operatorname{AbD}\left(24 \mathrm{~h}, 4{ }^{\circ} \mathrm{C}\right)$. Specimens were incubated for $48 \mathrm{~h}$ in anti-rabbit $\mathrm{IgG}$ (1:100) conjugated to tetramethylrhodamine isothiocyanate (TRITC) for visualisation of binding sites. Specimens were washed again in $\mathrm{AbD}$, incubated for $24 \mathrm{~h}$ in phalloidinfluorescein isothiocyanate (FITC) (as a counterstain that binds F-actin) and, after a final wash in $\mathrm{AbD}$, were mounted in PBS/glycerol (1:9) containing 2.5\% 2,4-diazabicylco 2.2.2 octane for confocal microscopic examination (Leica TCS-NT; Leica Microsystems, Milton Keynes, UK). Immunocytochemical controls included omission of primary antiserum and replacement of the primary antiserum with pre-immune serum.

\section{Results}

3.1. Classification of the schistosome aminopeptidase based on primary sequence structure

By searching the public databases with the peptide sequence NTDAEGRL that represents the conserved active site region of aminopeptidases (Kim and Lipscomb, 1993; Strater and Lipscomb, 1998), we located a cDNA that encodes an S. mansoni aminopeptidase (GenBank U83906). 
Sequence alignment and phylogenetic analyses using the amino acid sequence deduced from this cDNA revealed that the enzyme is a member of the M17 family of LAPs (Strater and Lipscomb, 1998). The $S$. mansoni LAP sequence is most closely related to an $S$. japonicum expressed sequence tag (EST) sequence (accession no. AF300423); the two proteins share $85 \%$ amino acid identity suggesting that they are orthologous enzymes (i.e. they perform the same function in these two discrete, schistosome species). The recent appearance in GenBank of $>40,000$ cDNAs, which may include the complete transcriptome of the adult stage of S. japonicum (Hu et al., 2003), enabled us to re-interrogate the public databases with accession nos. U83906 and AF300423. Although this analysis located over 80 additional entries encoding partial $S$. japonicum LAPs (not shown), each of these showed complete identity to the original EST sequence. This in turn supported the notion that a single copy gene encodes the LAP characterised here.

The relationship between the $S$. mansoni and $S$. japonicum LAPs and other members of the M17 family was investigated by comparing the sequence similarities in the conserved C-terminal domain (see below). The schistosome LAPs are most similar to an uncharacterised insect LAPs of Aedes gambiae (accession no. XP_311765) and Drosophila melanogaster (accession no. NP_650318). Remarkably, only one helminth LAP sequence with close similarity to the schistosome LAP was detected in the databases; this sequence is one of two LAP genes expressed by the nematode Caenorhabditis elegans (accession no. Q27245; 41 and 39\% amino acid identity to the S. mansoni and $S$. japonicum LAPs, respectively). The more diverged C. elegans LAP (accession no. P34629; 12 and 11\% amino acid identity, respectively, does not seem to have an orthologue in the schistosome genome, but resides in the clade that includes LAPs from several species of Leishmania. LAP members of the M17 family from the flowering plants Arabidopsis thaliana, Lycopersicon esculentum and Solanum tuberosum form a discrete and more distant clade from the schistosome LAPs (Fig. 1).

An alignment of the primary sequence of $S$. mansoni and $S$. japonicum LAPs with the A. gambiae, D. melanogaster and $C$. elegans sequences is presented in Fig. 2. The amino acids of the five sequences were $22.18 \%$ identical, $18.11 \%$ strongly similar and $11.65 \%$ weakly similar (total overall similarity 52\%). LAPs consist of a two-domain structure, a less conserved $\mathrm{N}$-terminal domain and a more conserved $\mathrm{C}$ terminal domain that contains the catalytic residues. As expected, the schistosome and other sequences show greater conservation in this catalytic C-terminal domain (Fig. 2). LAP are metallo-proteases and require the binding of two zinc ions that are pentahedrally coordinated within each active site. These metal ions act as nucleophiles and are essential for enzymatic activity. Accordingly, the residues that bind these zinc ions are highly conserved between all members of the M17 family of LAP enzymes including the schistosome LAPs. Residues Asp 289, Asp 367 and Glu 369 bind zinc 1, while Asp 289, Lys 284, Asp 307 and Glu 369 bind zinc 2. The residues Lys 296 and Arg 371 are also involved in the catalytic mechanism by acting as an electrophile and proton donor, respectively, and are thus also conserved in all LAPs.

\subsection{Functional expression of recombinant $S$. mansoni LAP in insect cells}

The cDNA sequences encoding the $S$. mansoni (U83906) and $S$. japonicum (AF300423) LAPs both lack start codons and are therefore incomplete. However, by analysing the more recent $S$. japonicum sequence entries (Hu et al., 2003), we completed the $5^{\prime}$ end of the LAP gene of this species and identified the start methionine. The protein sequence of the S. japonicum LAP (AF300423) was missing $26 \mathrm{NH}_{2^{-}}$ terminal amino acids (see accession no. BU795916 for $5^{\prime}$ end of S. japonicum cDNA at http://schistosoma.chgc.sh. $\mathrm{cn})$. By comparison, we found that the orthologue in S. mansoni LAP (U83906) was apparently missing only one amino acid, the start methionine. Accordingly, to express enzymatically active $S$. mansoni LAP in insect cells, we introduced a start codon (ATG) at the $5^{\prime}$ end of the $S$. manoni cDNA by incorporating it into the forward primer used in the amplification of the gene from the cDNA library. (However, screening of the $S$. mansoni EST data recently published by Verjovski-Almeida et al. (2003) revealed that the $S$. mansoni sequence was missing three $\mathrm{NH}_{2}$-terminal amino acids—see accession nos. MS1-0068T and MS10128T).

S. mansoni LAP was purified from recombinant baculovirus-infected insect cell extracts by single-step affinity chromatography on Ni-NTA agarose and was resolved as a $57.5 \mathrm{kDa}$ band following SDS-PAGE. The predicted molecular weight of the protein is $56,551.3 \mathrm{Da}$; the additional molecular weight of the recombinant protein is contributed by additional amino acids associated with the vector and the $\mathrm{His}_{6}$ tag (Fig. 3A). Purified recombinant $S$. mansoni LAP exhibited a specific activity of $1302 \mathrm{U} \mathrm{mg}^{-1}$ protein against the diagnostic LAP substrate, L-Leu-NHMec. In contrast, the specific activity of the LAP in the soluble extracts of adult $S$. mansoni and $S$. japonicum, was $1.4 \mathrm{U} \mathrm{mg}^{-1}$ protein and $1.45 \mathrm{U} \mathrm{mg}^{-1}$ protein, respectively.

Recombinant $S$. mansoni LAP was resolved by native polyacrylamide gels alongside samples of soluble $S$. mansoni extract, soluble $S$. japonicum extract and porcine kidney LAP (Fig. 3B). The gels were incubated in the substrate L-leu-NHMec for 10 min to detect LAP activity. A single band representing LAP activity was visualised under UV light in the recombinant LAP sample and this comigrated with a single band of activity detected in the S. mansoni and S. japonicum extracts. The positive control, porcine kidney LAP, was also visualised as a single band although this enzyme migrated further into the gel. 


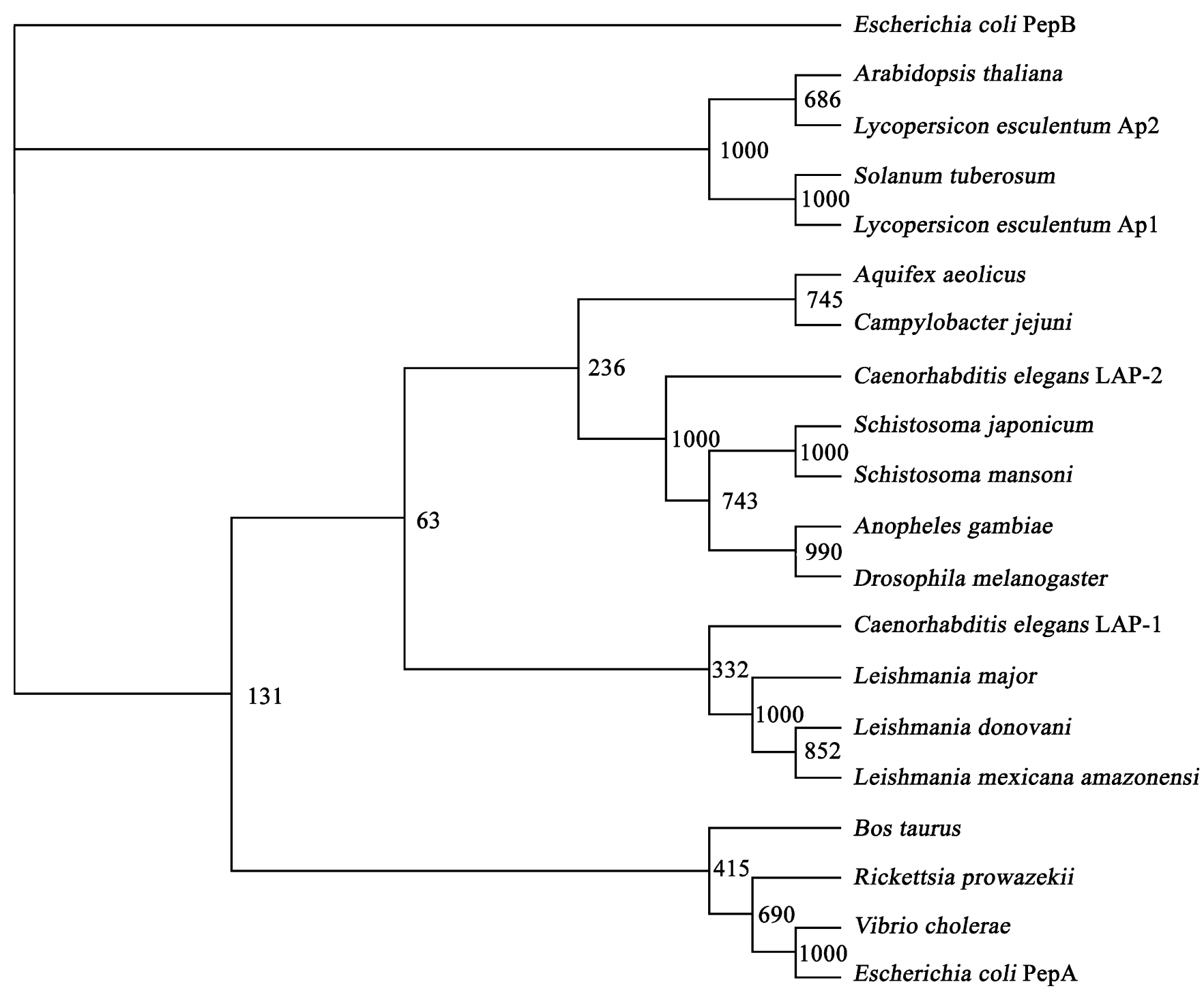

Fig. 1. The phylogenetic relationship between S. mansoni and S. japonicum LAP and various other members of the M17 family. Schistosoma mansoni LAP is most closely related to $S$. japonicum LAP. In turn the schistosome LAPs are most similar to the insect LAPs from the A. gambiae genome sequencing project (accession no. XP_311765) and D. melanogaster (accession no. NP_650318). Of the two LAP genes expressed by C. elegans, the schistosome LAPs are most similar to LAP-2 (accession no. Q27245). Caenorhabditis elegans LAP-1 resides in a clade that includes the LAP genes of the protozoan parasitic Leishmania species, Leishmania major (accession no. AF424693), Leishmania donovani (accession no. AF424692) and Leishmania mexicana amazonensis (accession no. AF424691). Plant LAP members of the M17 family A. thaliana (accession no. P30184), Solanum tuberosum (accession no. P31427), L. esculentum Ap-1 (accession no. Q10712) and L. esculentum Ap-2 (accession no. Q42876) form a discrete and more distinct clade from the schistosome LAP genes.

\subsection{Immunological detection of S. mansoni LAP in parasite extracts}

Rabbit antiserum prepared against recombinant S. mansoni LAP detected a single polypeptide in $S$. mansoni extracts that co-migrated at $\sim 57.5 \mathrm{kDa}$ with the recombinant SmLAP (Fig. 4A). A single protein was also detected in $S$. japonicum extracts but this migrated faster, at approximately $52 \mathrm{kDa}$. LAP is also expressed in $S$. mansoni cercariae and it migrated as a similar sized protein to the adult form (Fig. 4B).

\subsection{Physico-chemical properties of recombinant S. mansoni LAP}

Recombinant $S$. mansoni LAP activity displayed a preference for a neutral/slightly alkaline ( $\mathrm{pH}$ 6.5-9.4) environment. Enzyme activity was optimal at $\mathrm{pH} 8.25$ and was not detectable below pH 6.5 (Fig. 5A). This $\mathrm{pH}$ profile correlated closely with the profile for LAP activity in soluble extracts of $S$. mansoni and $S$. japonicum that also exhibited optimal activity at $\mathrm{pH}$ 8.25. LAP members of the M17 family are metalloenzymes and thus require 
SmLAP

SjLAP AgLAP DMLAP CELAP-2

SMLAP

SjLAP AgLAP DMLAP CELAP-2

SmLAP

SjLAP

AgLAP

DMLAP

CELAP-2

SmLAP

SjLAP AgLAP DMLAP CELAP-2

SMLAP

SjLAP

AgLAP

DMLAP

CeLAP-2

SmLAP

SjLAP

AgLAP

DMLAP

CELAP-2

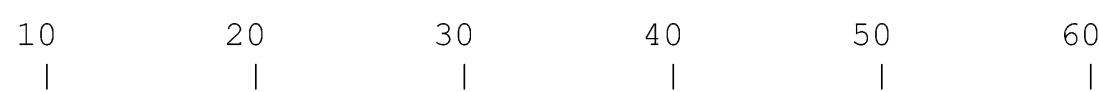
-MSTSVVTPVEP-VSCLTDPSYD----AVLLLNDDIEHLPRDLQLAYKPLKEFSEVNPKE ---MSLVTPVEP-VFNLVDSAYD----AVLLLNDDVEHLPNALQLALNALKEFSEVNPKE --VPSYLPCEID-PVDNLEVALNRAARCDTLCVIDDANLHPVLAREIEQQKTFDAG---I MGIEKILPCTLR-LNKLMSLAGN-----DCLCI I RRNAVPNELKATFEEHRKFDKS---E MSLQSLLSTKIVRATS IADAAFDAVVLVGSQDNVQQFGAIQQVSAIAPAVNNFLKLHSGA
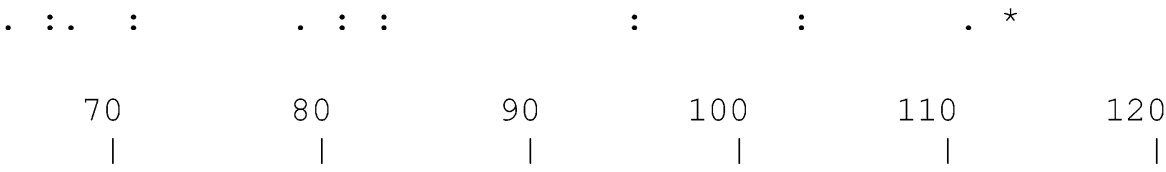
SDEVSVIPFPEHPSKR--LIESPTGRLNTDEADIRNVYDAAVNGMLKLLKIGCKS PLLCC SDEVSIIPFPQHPSKR--LIFSPTGQLNTDEADIRNGYDAAVNGMLKLLKIGCKSPLLCC CSEVSCFRCTVGSEPVR-VVYSPVGELS-DFDDVRRYAEAAGQAMRRALKAGAKFPLLVL DSSISCFKVPNVDQPV---VYAPVSELT-DYDDVRSYQEAAKRSMEKVLKAGFHTPLLFV FNSTSLVQVDSSVVPSGRLILSGTGNVSRDYDDVRRYQAAARKGISMALSAGVKSPLLIT

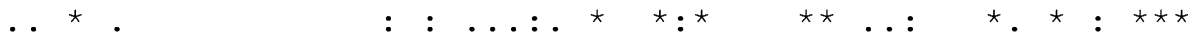

$\begin{array}{rrrrrr}130 & 140 & 150 & 160 & 170 & 180 \\ \mid & \mid & \mid & \mid & \mid\end{array}$
GSFISAPKDFQWCERNCLLLNAILGAYHVLYTPLEVREMLPNKFPKAIRFGVMEAD---GSFVSTPKDAQWAQRNCLLLNAVLGAYHALYT PLEVREMLPKKF PKAIREGVMEAN---PAPTERFSAAPLVA-----L---LGALHELYVPLQLREDVPAKRQRYAKIGLLHPDGAQR PK-VKREPEVELCT-----V---LGALEQLYVPIQLREAGTLKDPRVTTLSVQIDD--PR LP-NSREPNAELVA--------ALGALTPVYTPLNVREEENKQ--KLNQLGLLAIGNSDT $\star \star \star \quad:{ }^{\star}{ }^{\star}:::^{\star} \quad: \quad: \quad:$ : .

$\begin{array}{rrrrrr}190 & 200 & 210 & 220 & 230 & 240 \\ \mid & \mid & \mid & \mid & \mid\end{array}$

-NSLLNIANAIEEGRTLARDIGGSDPERMAAPKIAEYLQNALNG--IKGVTVTVDKVDAK -DSLLNIAKAIEEGRTLARDIGGSDSERMAAPKIAEYLKNALDG--LTGVTITINKVDAK GQDLLRQVQSLEAGRFVARDIGGGDPERMAP PRVAEYVLQAFAGGPVKVTVVDDPRVFER AEA I FQEAL I LEAGR FVARD I GVGDPERMT P IQVEKY IKPLFD--KLNVNVISDTQVLQK SARLEKLVEAYDAS FTVCRDVGEAGPERMAP PRVAEYIQGAFANGNIKVTVVDDQSVILK

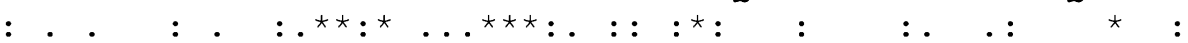

$$
\begin{aligned}
& \begin{array}{lllll}
250 & 260 & 270 & 280 & 290
\end{array}
\end{aligned}
$$
KYPLMAAVNRAASVVPRHDGRVVHVDYKPSNSKEIDTSLFLVGKGITFDTGGADVKAGGI
KYPLMAAVNRAASVVPRHDGRVVHIDYKPSSSKEIDTSLFLVGKGITFDTGGADVKAGGI

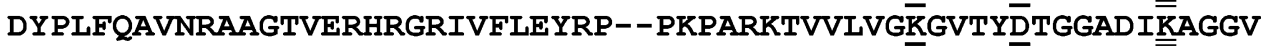

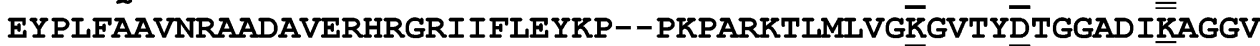
DFPLMAAVNRAANCVKEHQARLIRLEYVG--EGETQDTFFVVGKKGVTIDTGGCDLKKTGGH

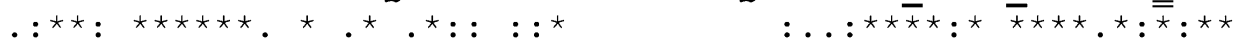

$$
\begin{array}{llllll}
310 & 320 & 330 & 340 & 350 & 360
\end{array}
$$

MAGMHRDKCGAAAIAGLMKTIGLLQPDGISVSAGLAFVRNSIGSESYVADEIIIARSGQR MAGMHRDKCGAAAIRGLMKT IGLLQPTGLSDSAGLAFVRNSGGEESYVADEI I IARSGQR MAGMSRDKCGAAAVAGFMKVVEQRQPQDVHAIGVLCMVRNSVGEECYVSDEMVTSRAGVR MAGMSRD KCGAAAVAGFMQVVSQLQPDDIHVVAALCMVRNSVGEECYVADEVITSRAGLH MFGMCRD KYGSAVVGGFFKAIDVLKPKNIKAVGYMCMVRNSIGSHAYTCDEVITSRSGKR

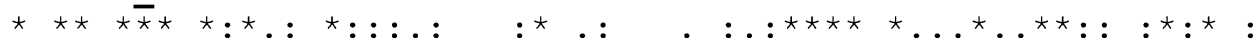

Fig. 2. 


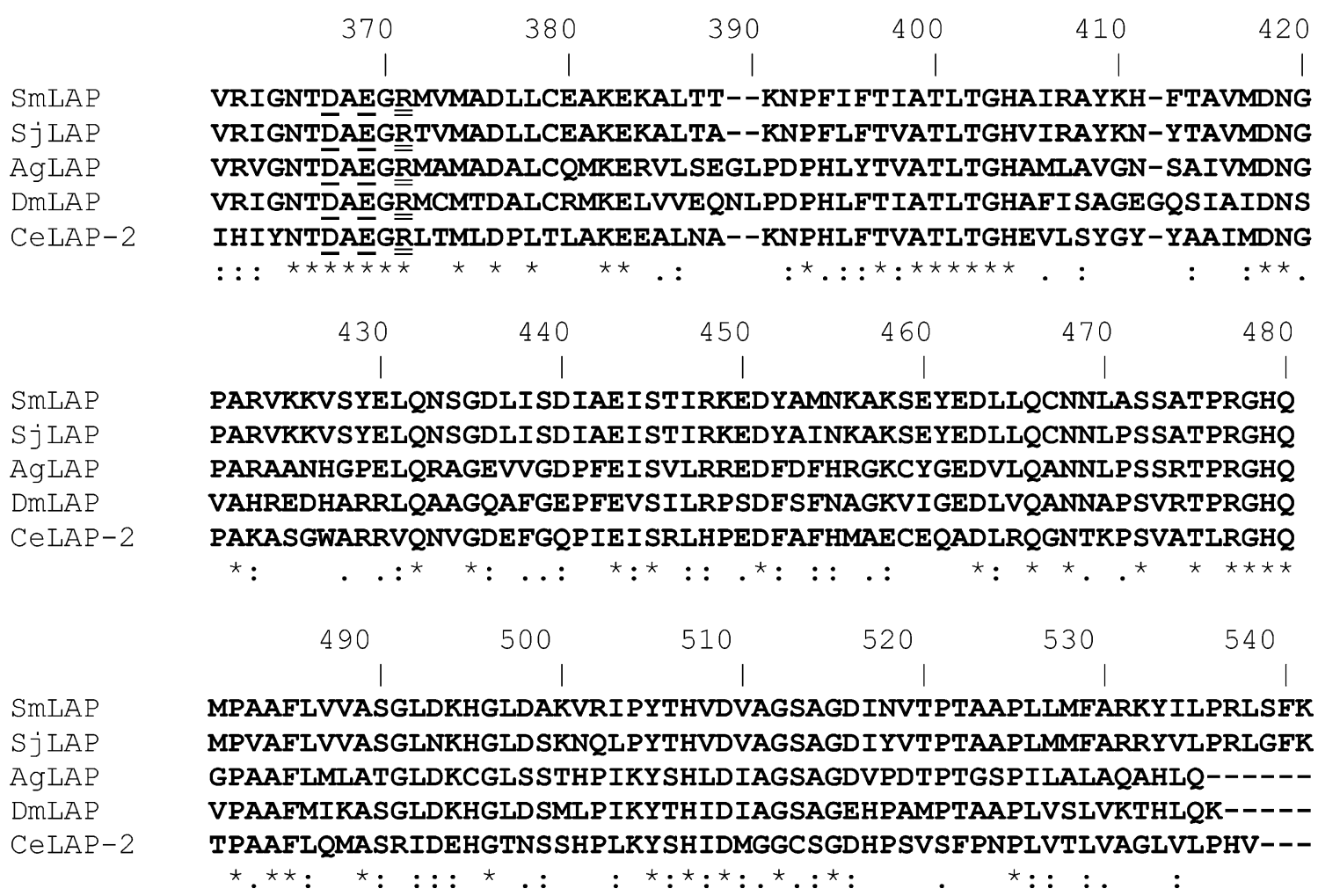

Fig. 2. Alignment of LAP from S. mansoni (SmLAP) with S. japonicum LAP (SjLAP), Anopheles gambiae LAP (AgLAP, accession no. XP_311765), D. melanogaster LAP (DmLAP, accession no. NP_650318) and LAP-2 from C. elegans (CeLAP-2, accession no. Q27245). This alignment indicates the conserved sites $(*)$ among the five sequences $(22.18 \%$ identical). Of the sequences, $18.11 \%$ are strongly similar (:) and $11.65 \%$ are weakly similar (.). All five sequences are of similar length (SmLAP, 523 residues; SjLAP, 521 residues; AgLAP, 516 residues; DmLAP, 508 residues; CeLAP, 522 residues), although it must be noted that AgLAP is incomplete at the N-terminus. Each LAP subunit is a two-domain structure, consisting of an N-terminal domain and a catalytic Cterminal domain. The sequences are more conserved in the catalytic C-terminal domain of each subunit (highlighted in bold). Active LAP requires the binding of two zinc ions that are pentahedrally coordinated within each active site. Zinc 1 is bound by Asp 289, Asp 367 and Glu 369 (underlined). Zinc 2 is bound by Asp 289, Lys 284, Asp 307 and Glu 369 (underlined). These residues are highly conserved between all members of the M17 family of LAP enzymes. Lys 296 and Arg 371 (double underlined) are also involved in catalysis acting as an electrophile and proton donor, respectively.

the presence of metal cations to maintain enzyme activity and stability (Kim and Lipscomb, 1993; Taylor, 1993; Strater and Lipscomb, 1998). An analysis of the metal ion requirement for $S$. mansoni LAP showed that manganese and magnesium ions enhanced recombinant LAP activity 2-4 fold. LAP activity in soluble extracts of S. mansoni and S. japonicum was likewise enhanced by the addition of these metal ions. In contrast, zinc cations reduced the activity of recombinant LAP, $S$. mansoni and $S$. japonicum by as much as 94,84 and $31 \%$, respectively. The metal chelators EDTA and 1,10-phenanthroline and the aminopeptidase inhibitor bestatin were all inhibitory for both recombinant and native schistosome LAP (Table 1).

Recombinant $S$. mansoni LAP exhibited a marked preference for Leu when compared to Ala, Tyr and Pro in keeping with its classification as a M17 LAP (Fig. 5B). The LAP activity in S. mansoni and S. japonicum soluble extracts shows a similar substrate profile. Porcine kidney LAP also demonstrated a preference for Leu but its relative activity against Ala and Tyr differed from the schistosome enzyme. Characteristically, these aminopeptidases do not cleave the bulky hydrophobic residue Pro (Kim and Lipscomb, 1993; Taylor, 1993; Strater and Lipscomb, 1998).

\subsection{Immunolocalisation of S. mansoni LAP by confocal scanning laser microscopy}

LAP-immunoreactivity was predominately associated with the alimentary tract. Strong immunoreactivity surrounded the subapical mouth, with staining localised below the musculature of the oral sucker (Fig. 6A); no staining was associated with the ventral sucker (Fig. 6B). Within the alimentary tract itself, the oesophagus was immunonegative while strong staining was observed immediately distal to the oesophageal opening of the paired gut caeca (Fig. 6B and C). LAP-immunoreactivity was associated with the syncitial gastrodermis and filled the lumen of the anterior regions of the paired gut caeca (Fig. 6B-D). Staining within the lumen and in the gastrodermis decreased posteriorly becoming undetectable in the distal third of the worm (Fig. 6E). LAPimmunoreactivity was considerably stronger within the gut 
$\mathbf{A}$

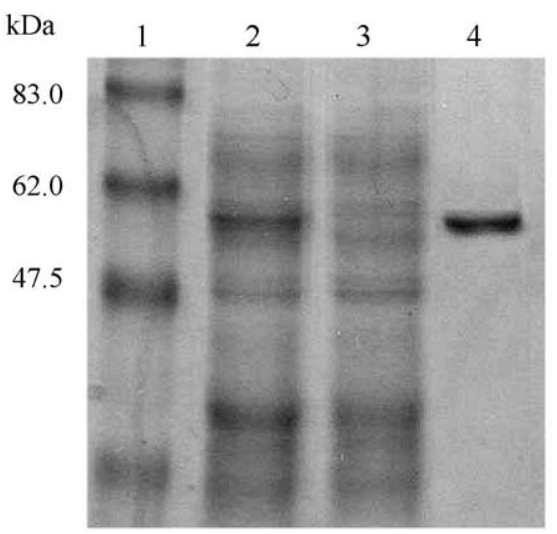

B

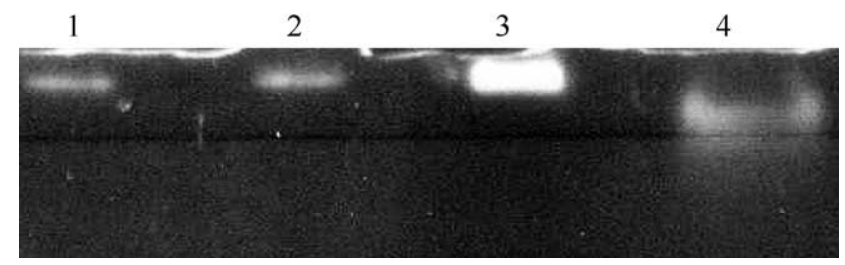

Fig. 3. Purification of functionally active recombinant $S$. mansoni LAP expressed in insect cells. (A) Samples from the purification were analysed on $12 \%$ reducing SDS-PAGE. Lane 1, protein molecular size markers; Lane 2, soluble extract of insect cells infected with recombinant baculovirus encoding SmLAP; Lane 3, soluble extract of uninfected insect cells; Lane 4, Ni NTA-agarose-purified recombinant SmLAP $(0.7 \mu \mathrm{g})$. (B) Soluble extracts of adult schistosome and recombinant SmLAP were separated in $10 \%$ native PAGE and subjected to direct fluorogenic substrate analysis by probing with L-leu-NHMec. Lane 1, soluble extracts of $S$. mansoni; Lane 2, soluble extracts of S. japonicum; Lane 3, purified recombinant SmLAP; Lane 4, porcine kidney LAP.

of females than males. A distinct layer of sub-tegumental immunostaining that became progressively more intense posteriorly was observed along the length of the body (Fig. 6E and F). In contrast to LAP immunostaining in the gut, the sub-tegumental staining was stronger in males than females. No staining was observed in the reproductive system. All controls gave negative results (Fig. 6D, inset).

\section{Discussion}

Sequence alignments and phylogenetic analyses revealed that the schistosome aminopeptidases encoded by the cDNAs described were members of the clan MF that contains only one family, M17, also known as the leucyl aminopeptidases (LAPs) (Strater and Lipscomb, 1998). Whereas LAPs have been identified in many organisms, to date no M17 aminopeptidase has been characterised from a parasitic worm. Aminopeptidase activity, however, has been detected in parasite extracts from schistosomes
A

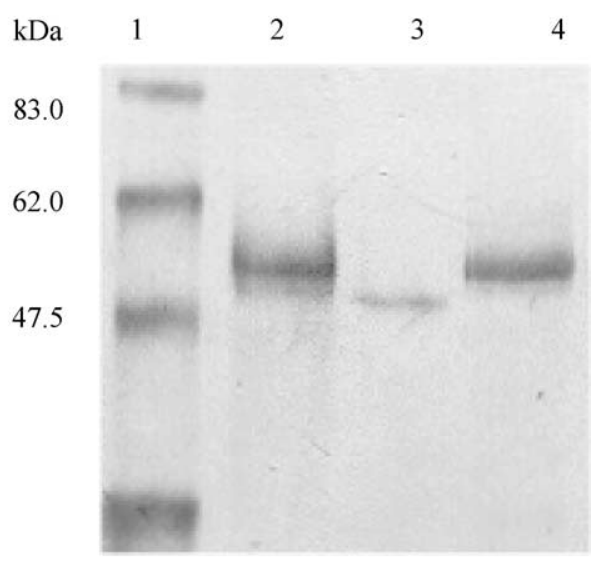

B

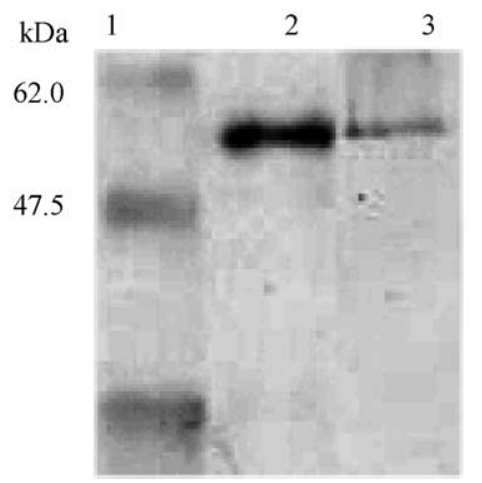

Fig. 4. Immunoblot identification of schistosome LAP in parasite extracts. Somatic extracts from $S$. japonicum, S. mansoni and recombinant LAP were resolved using $12 \%$ SDS-PAGE, blotted to nitrocellulose membrane and probed with polyclonal antibodies prepared against $S$. mansoni recombinant LAP. (A) Lane 1, molecular size markers; Lane 2, soluble extracts of adult S. mansoni; Lane 3, soluble extracts of adult S. japonicum; Lane 4, purified recombinant SmLAP. (B) Lane 1, molecular size markers; Lane 2, soluble extracts of adult $S$. mansoni; Lane 3, soluble extracts of $S$. mansoni cercaria. Pre-immune rabbit sera did not bind to any proteins in parasite extracts (not shown).

(Auriault et al., 1982; Damonneville et al., 1982; Xu and Dresden, 1986; Xu et al., 1988, 1990; Doenhoff et al., 1988), Fasciola hepatica (Acosta et al., 1998; Piacenza et al., 1999) and Ascaris suum (Rhoads and Fetterer, 1998). The well-characterised Haemonchus contortus aminopeptidase, H11 (accession no. AJ249941) (Newton, 1995), differs from the aminopeptidases investigated here in that it is a membrane bound, microsomal enzyme of Clan MA(E), Family M1. The schistosome LAPs are most closely related to LAPs of the mosquito A. gambiae and the fruitfly D. melanogaster, but the function of these enzymes is unknown. More interestingly, we found only one helminth sequence in the public databases that was related to the schistosome LAP, namely one of the two LAPs of the freeliving nematode C. elegans, accession no. Q27245, but this enzyme is also uncharacterised. Schistosomes apparently do 
A

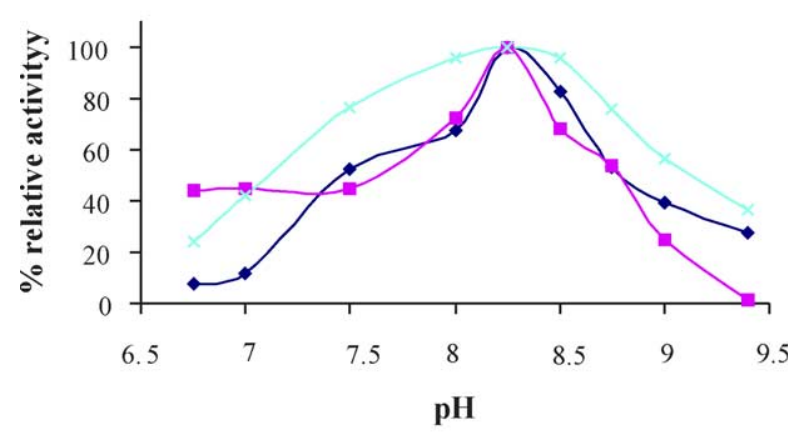

B

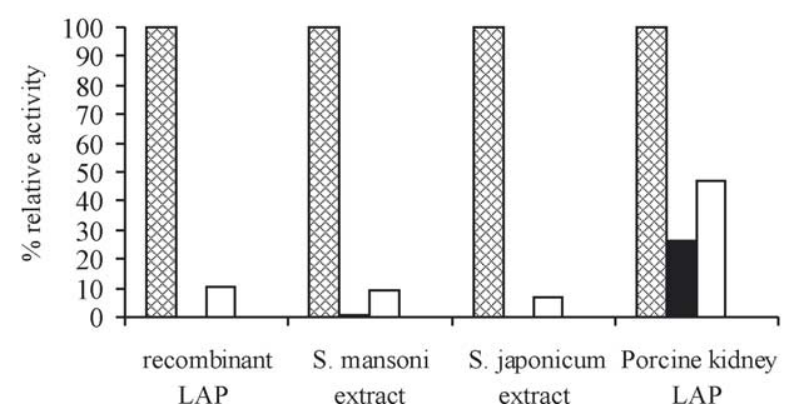

Fig. 5. Comparison of the biochemical properties of recombinant SmLAP with the LAP activity in soluble extracts of adult schistosomes. (A) The effect of $\mathrm{pH}$ on LAP activity of purified recombinant SmLAP (x) soluble extracts of adult $S$. mansoni $(\bullet)$ and soluble extracts of adult $S$. japonicum (ם). (B) The relative activity of recombinant SmLAP, soluble extracts of adult $S$. mansoni, soluble extracts of adult $S$. japonicum and porcine kidney against the fluorogenic substrates L-leu-NHMec (hatched bar), L-alaNHMec (black bar), L-tyr-NHMec (white bar) and L-pro-NHMec (no activity). The hydrolysis of substrates was measured as a percentage of the optimal substrate L-leu-NHMec.

not express the orthologue of the second aminopeptidase (P34629) of C. elegans.

LAPs consist of two structurally unrelated domains. The $\mathrm{NH}_{2}$-terminal domain is not conserved amongst LAPs, varies in length among members, and does not exhibit identity with other proteins. In contrast, the $\mathrm{COOH}$-terminal domain contains the catalytic and the zinc-binding residues and is structurally related to carboxypeptidases $\mathrm{A}$ and $\mathrm{T}$ (Clan MC) (Kim and Lipscomb, 1993; Taylor, 1993; Strater and Lipscomb, 1998). The substrate and zinc binding sites are well conserved in the M17 family and consequently its members from prokaryotes, plants and animals display similar activity profiles (Strater and Lipscomb, 1998). These sites are conserved in $S$. mansoni and $S$. japonicum LAPs, and recombinant $S$. mansoni LAP displayed biochemical properties consistent with those of other M17 family peptidases. Specifically, recombinant $S$. mansoni LAP had a marked substrate preference for $\mathrm{NH}_{2}$-terminal leucine residues, a requirement for metal ions for activity and a neutral to weakly alkaline $\mathrm{pH}$ optimum.

By fluorogenic substrate gel analysis, we detected a single aminopeptidase activity in extracts of adult $S$. mansoni and $S$. japonicum suggesting that the enzyme is the major aminopeptidase activity in these preparations. In support of this, recombinant SmLAP exhibited a similar biochemical profile to the LAP activity in the parasite extracts. Using antibodies prepared against the recombinant SmLAP we identified the enzyme as a single protein in soluble extracts of mixed adult $S$. mansoni at $\sim 57.5 \mathrm{kDa}$, which is slightly higher than its predicted molecular mass of $56,739.4 \mathrm{kDa}$ from analysis of the primary sequence. The $S$. japonicum LAP also resolved as a single protein, but migrated at approximately $52 \mathrm{kDa}$ which is lower than its predicted mass of $56,210.68 \mathrm{kDa}$. The enzyme of each schistosome, however, co-migrated as single bands in native polyacrylamide gels and their specific activities in the soluble extracts were similar ( $S$. mansoni LAP $1.4 \mathrm{U} \mathrm{mg}^{-1}$ protein and S. japonicum LAP $1.45 \mathrm{U} \mathrm{mg}^{-1}$ protein). Disparities in the predicted and apparent molecular sizes of the $S$. mansoni LAP may be due to post-translational glycosylation which would tend to cause proteins to migrate slower in gels but this

Table 1

Effect of divalent metal cations, chelators and inhibitors on recombinant $S$. mansoni leucine aminopeptidase (LAP) and LAP activities in soluble extracts of S. mansoni and S. japonicum

\begin{tabular}{|c|c|c|c|}
\hline & Recombinant $S$. mansoni LAP & S. mansoni soluble extract & S. japonicum soluble extract \\
\hline Control (no treatment) & 100 & 100 & 100 \\
\hline $\mathrm{MnCl}_{2}(0.05 \mathrm{mM})$ & $139.8 \pm 0.5$ & $380.4 \pm 1.5$ & $442.1 \pm 2.9$ \\
\hline $\mathrm{MnCl}_{2}(0.5 \mathrm{mM})$ & $87.6 \pm 1.9$ & $236.4 \pm 0.2$ & $364.6 \pm 1.6$ \\
\hline $\mathrm{MgCl}_{2}(0.05 \mathrm{mM})$ & $186.4 \pm 1.5$ & $299.7 \pm 1.6$ & $146.3 \pm 5.6$ \\
\hline $\mathrm{ZnCl}_{2}(0.05 \mathrm{mM})$ & $24.7 \pm 0.3$ & $29.1 \pm 0.1$ & $88.9 \pm 4.9$ \\
\hline $\mathrm{ZnCl}_{2}(0.5 \mathrm{mM})$ & $6.0 \pm 0.5$ & $15.6 \pm 0.1$ & $69.4 \pm 0.6$ \\
\hline \multicolumn{4}{|l|}{ Metal chelators } \\
\hline EDTA $(5 \mathrm{mM})$ & $51.5 \pm 0.6$ & $36.7 \pm 0.7$ & $101.9 \pm 1.5$ \\
\hline$o$-Phenanthroline $(5 \mathrm{mM})$ & $28.6 \pm 0.6$ & $56.4 \pm 0.2$ & $38.5 \pm 0.6$ \\
\hline \multicolumn{4}{|l|}{ Inhibitor } \\
\hline Bestatin $(50 \mu \mathrm{M})$ & $<0.05$ & $<0.05$ & $30.2 \pm 0.1$ \\
\hline
\end{tabular}




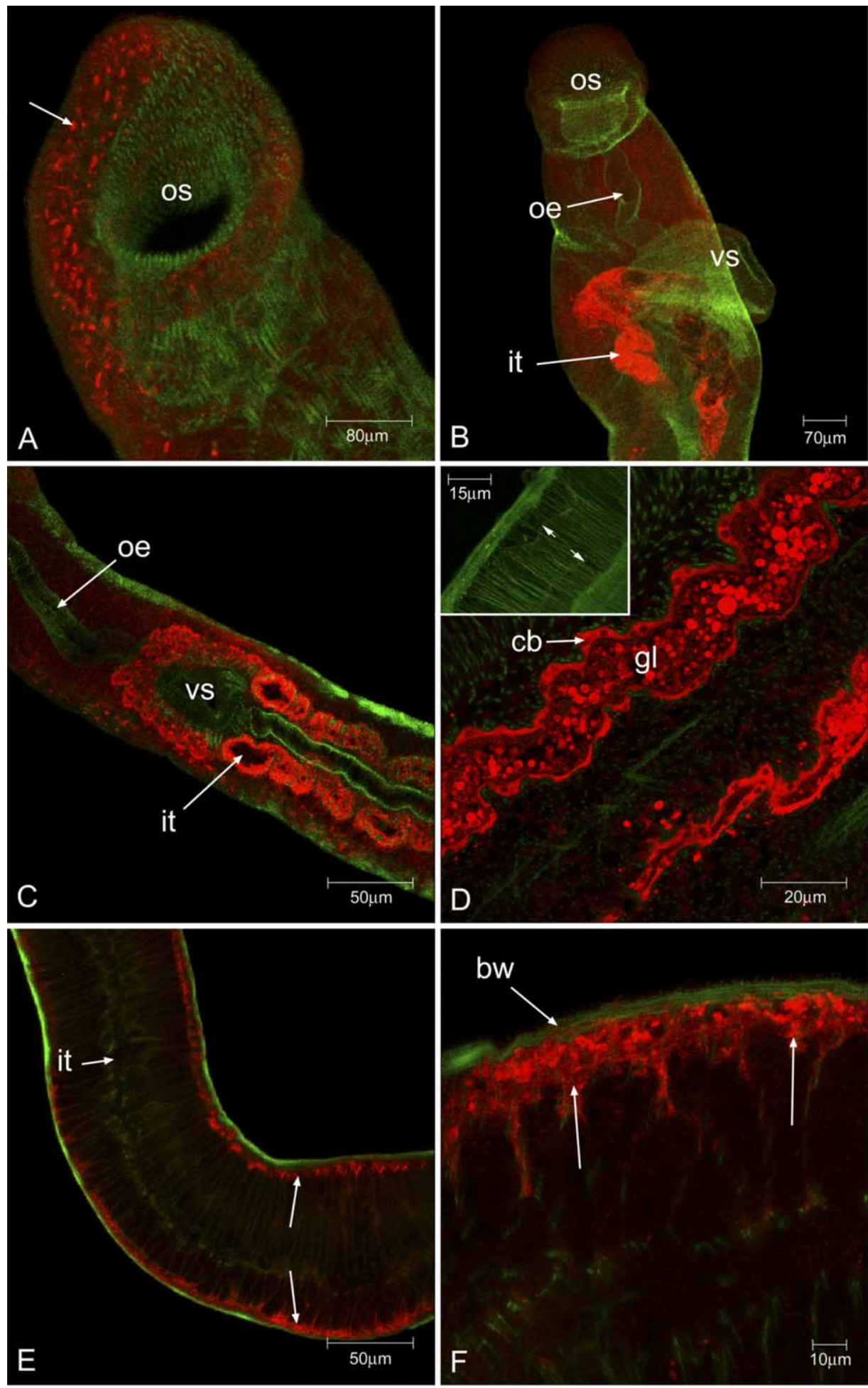

Fig. 6. Confocal scanning laser micrographs of $S$. mansoni immunostained for LAP (red) and counterstained for filamentous actin with phalloidin-FITC (green). (A) Anterior of male showing pulses of LAP-immunoreactivity (arrow) lying beneath the musculature of the oral sucker (os). (B) Feeding and attachment apparatus of male illustrating the lack of immunostaining in the oesophagus (oe) in contrast to the strong staining in the paired limbs of the intestine (it). No staining is associated with the ventral sucker (vs). os, oral sucker. (C) Intense immunoreactivity invests the intestinal caeca (it) of this female with a clear demarcation in staining between the intestine and the oesophagus (oe). vs, ventral sucker. For the most part immunoreactivity to LAP was considerably stronger in the gut of females than males. (D) Higher powered image of the intestine with strong immunostaining in the gut lumen (gl) and syncytial cell bodies (cb) of the gastrodermis. Insert: control specimen (showing region of gynaecophoric canal) stained with pre-immune serum illustrating an absence of unspecific staining within the gut (arrows). (E) LAP-immunoreactivity becomes progressively weaker in the intestine (it) moving posteriorly, while sub-tegumental staining (arrows) increases in intensity. (F) High powered image of immunoreactivity (arrows) lying below the body wall musculature (bw) in the posterior third of a male. This staining lying beneath the tegument/body wall was noted to be stronger in males than in females. 
would not account for the faster migrating $S$. japonicum LAP. Isoelectric focusing techniques resolved three $S$. mansoni aminopeptidase activities (Damonneville et al., 1982) that may result from heterogeneity in the levels of glycosylation. The LAPs of $S$. mansoni and the $S$. japonicum both possess one potential $N$-glycosylation site, Asp-497 in S. mansoni LAP and Asp-166 in the $S$. japonicum orthologue.

The $S$. mansoni LAP was predominantly expressed by gastrodermal cells lining the caecum where digestion of host haemoglobin takes place following the lysis of host red blood cells in the oesophagus. This localisation implies that LAP is involved in this process of digestion, and the greater intensity of staining in female worms compared with males correlates with the higher consumption of red blood cells in the former (Brindley et al., 1997; Tort et al., 1999). However, $\mathrm{pH}$ profile studies showed that the recombinant and native LAPs are active in the $\mathrm{pH}$ range 6.5-9.0 (optimal at $\mathrm{pH} 8.25$ ) and inactive below $\mathrm{pH} 6.5$ which suggest that the enzyme may not function efficiently in the slightly acidic environment of the gut lumen. Endopeptidases known to be involved in hemoglobin catabolism, including cathepsin L1, cathepsin L2 and cathepsin D, are all optimally active at low pH (<5.0) (Brindley et al., 1997; Tort et al., 1999) while cathepsin $\mathrm{B} 1$ is optimally active at $\mathrm{pH} \sim 6.5$ (Sajid et al., 2003). In addition, the exopeptidase, cathepsin C (dipeptidylpeptidase I) is optimally active at $\mathrm{pH}$ 4.0-5.0 (Hola-Jamriska et al., 1998, 1999, 2000). In contrast to all these peptidases, the SmLAP does not possess a signal peptide necessary for passage into the secretory pathway and therefore the enzyme most likely functions intracellularly. These observations allow us to hypothesise on the final stages of hemoglobin digestion in our model-short peptides generated by the action of various endopeptidases in the gut lumen are transported into the cytosol of gastrodermal cells surrounding the gut lumen where they are hydrolysed to free amino acids by LAP. Free amino acids could subsequently distribute to various schistosome tissues by simple diffusion or via specific amino acid permeases (Dalton et al., 2004).

SmLAP was localised to other schistosome tissues, including sub-tegumental regions, suggesting that the enzyme plays an additional role(s) in schistosome biology. The specific role of LAPs in plant and animal tissues is not known but their expression in different tissues is consistent with their role in a variety of processes such as the maturation of proteins, the terminal degradation of proteins and the metabolism of secreted regulatory molecules (Strater and Lipscomb, 1998). Since they are exopeptidases, they must function in degrading either biologically active small peptides, such as hormones, or large polypeptides in concert with endopeptidases (Strater and Lipscomb, 1998; Gavigan et al., 2001). In this context, it is of interest to note that cathepsin L1 (Dalton et al., 1996; Brady et al., 1999a,b) and cathepsin B2 (Caffrey et al., 2002) endoproteases have also been localised to the tegument and hence it is plausible that a multi-enzymatic degradation process, similar to that described for hemoglobin digestion in the gut, also occurs in this tissue. The up-regulation of tomato LAP expression is associated with mechanical wounding or infestation and may be part of a protein turnover or tissue repair mechanism (Gu et al., 1999). Given that the schistosome surface membrane is in constant flux, it is possible that cathepsin L1, cathepsin B2 and LAP work in concert in the dismantling, degradation and turnover of the protein components of the membrane.

SmLAP activity was also detected in soluble cercarial extracts, consistent with reports of LAP activity in schistosomula and adults (Auriault et al., 1982; Damonneville et al., 1982). The process of membrane turnover and repair is critical to the transformation of the infective freeliving cercaria to the schistosomule, and soon after skin penetration, the schistosomule begins to feed on host blood. Therefore, the function of the LAP in these juvenile stages of schistosomes may be similar to that in the adult worms, i.e. hemoglobin digestion and surface membrane re-modelling. Another function of LAP may be facilitating hatching since LAP activity was associated with the miracidial stage and hatching was prevented by the general aminopeptidase inhibitor bestatin. However, two LAP activities, one membrane-associated and one soluble, were detected in egg homogenates and hatching fluid (Xu and Dresden, 1986; Xu et al., 1988, 1990). The membrane-associated aminopeptidase was further characterised and shown to migrate as a single band at $140 \mathrm{kDa}$ following reducing SDS-PAGE (Xu et al., 1990) and is clearly not the enzyme described here. Accordingly, further work is required to elucidate whether the $57.5 \mathrm{kDa}$ LAP, a different soluble LAP or the $140 \mathrm{kDa}$ membrane-associated aminopeptidase, is responsible for miracidial hatching.

Finally, schistosome LAP is worthy of investigation as a vaccine candidate given its potentially crucial roles in parasite nutrition and maintenance of tegumental structure, and its expression in the early invasive stages of the parasite. Of relevance in this regard is the recent finding of significant protection in sheep against the related fluke parasite $F$. hepatica by vaccination with purified Fasciola LAP alone or in combination with a cathepsin L protease (Piacenza et al., 1999). Since the recombinant enzyme is available in active enzymatic conformation, the vaccine potential of the schistosome LAP can now be evaluated.

\section{Acknowledgements}

This work was supported by a joint North-South Cooperation grant from the HRB (Ireland) and the Research and Development Office (Northern Ireland), and by The Wellcome Trust, UK. PB is a recipient of a Burroughs Wellcome Fund Scholar Award in Molecular Parasitology 
and of an E.T.S. Walton Award from Science Foundation Ireland. We would like to thank James O. McInerney for help with the sequence alignments and phylogenetic tree construction.

\section{References}

Acosta, D., Goni, F., Carmona, C., 1998. Characterization and partial purification of a leucine aminopeptidase from Fasciola hepatica. J. Parasitol. 84, 1-7.

Auriault, C., Pierce, R., Cesari, I.M., Capron, A., 1982. Neutral protease activities at different developmental stages of Schistosoma mansoni in mammalian hosts. Comp. Biochem. Physiol. 72, 377-384

Brady, C.P., Brindley, P.J., Dowd, A.J., Dalton, J.P., 1999a. Schistosoma mansoni: differential expression of cathepsin L1 and cathepsin L2 suggests discrete biological functions for each enzyme. Exp. Parasitol. 94, 75-83.

Brady, C., Dowd, A., Brindley, P.J., Ryan, T., Day, S.R., Dalton, J.P., 1999b. Functional expression and localization of Schistosoma mansoni cathepsin L1 support its role in the degradation of host hemoglobin. Infect. Immun. 67, 368-374.

Brindley, P.J., Kalinna, B.H., Dalton, J.P., Day, S.R., Wong, J.Y., Smythe, M.L., McManus, D.P., 1997. Proteolytic degradation of host hemoglobin by schistosomes. Mol. Biochem. Parasitol. 89, 1-9.

Caffrey, C.R., Salter, J.P., Lucas, K.D., Kheim, D., Hseih, I., Lim, K.C., Ruppel, A., McKerrow, J.H., Sajid, M., 2002. SmCB2, a novel tegumental cathepsin B from adult Schistosoma mansoni. Mol. Biochem. Parasitol. 121, 49-61.

Curley, P., O’Donovan, S.M., McNally, J., Dalton, J.P., 1994. Aminopeptidase from Plasmodium berghei, Plasmodium chabaudi chabaudi and Plasmodium falciparum. J. Eukaryot. Microbiol. 41, 119-123.

Dalton, J.P., Clough, K.A., Jones, M.K., Brindley, P.J., 1996. Characterization of the cathepsin-like cysteine proteinases of Schistosoma mansoni. Infect. Immun. 64, 1328-1334.

Dalton, J.P., Skelly, P., Halton, D.W., 2004. Role of the tegument and gut in nutrient uptake by parasitic platyhelminths. Can. J. Zool. in press.

Damonneville, M., Auriault, C., Pierce, R.J., Capron, A., 1982. Antigenic properties of Schistosoma mansoni aminopeptidases: evolution during the development in mammalian hosts. Mol. Biochem. Parasitol. 6, $265-275$

Doenhoff, M.J., Modha, J., Curtis, R.H., Adeoye, G.O., 1988. Immunological identification of Schistosoma mansoni peptidases. Mol. Biochem. Parasitol. 31, 233-240.

Engels, D., Chitsulo, L., Montresor, A., Savioli, L., 2002. The global epidemiological situation of schistosomiasis and new approaches to control and research. Acta Trop. 82, 139-146.

Ennis, O., Corcoran, K., Kavanagh, K., Mahon, B.P., Doyle, S., 2001. Baculovirus expression of parvovirus B19 (B19V) NS1: utility in confirming recent infection. J. Clin. Virol. 22, 55-60.

Gavigan, C.S., Dalton, J.P., Bell, A., 2001. The role of aminopeptidases in haemoglobin degradation in Plasmodium falciparum-infected erythrocytes. Mol. Biochem. Parasitol. 117, 37-48.

Gu, Y.Q., Holzer, F.M., Walling, L.L., 1999. Overexpression, purification and biochemical characterization of the wound-induced leucine aminopeptidase of tomato. Eur. J. Biochem. 263, 726-735.

Hola-Jamriska, L., Tort, J.F., Dalton, J.P., Day, S.R., Fan, J., Aakov, J.G., Brindley, P.J., 1998. Schistosoma japonicum cathepsin C: cDNA encoding the proenzyme, biochemical analysis, and phylogenetic relationships. Eur. J. Biochem. 255, 527-534.

Hola-Jamriska, L., Dalton, J.P., Aaskov, J.G., Brindley, J.P., 1999. Dipeptidyl peptidase I and III activities in adult schistosomes. Parasitology 118, 275-282.

Hola-Jamriska, L., King, L.T., Dalton, J.P., Mann, V.H., Aaskov, J.G., Brindley, P.J., 2000. Functional expression of dipeptidyl peptidase 1 (cathepsin C) of the oriental blood fluke Schistosoma japonicum in Trichoplusia ni insect cells. Protein Expr. Purif. 19, 384-392.

Hu, W., Yan, Q., Shen, D.-K., Liu, F., Zhu, Z.D., Song, H.D., Xu, X.R., Wang, Z.J., Rong, Y.P., Zeng, L.C., Wu, J., Zhang, X., Wang, J.J., Xu, X.R., Wang, S.Y., Fu, G., Zhang, X.L., Wang, Z.Q., Brindley, P.J., McManus, D.P., Xue, C.L., Feng, Z., Chen, Z., Han, Z.G., 2003. Evolutionary and biomedical implications of a Schistosoma japonicum complementary DNA resource. Nat. Genet. 35, 139-147.

Kim, H., Lipscomb, W.N., 1993. Differentiation and identification of the two catalytic metal binding sites in bovine lens leucine aminopeptidase. Proc. Natl Acad. Sci. USA 90, 5006-5010.

Newton, S.E., 1995. Progress on vaccination against Haemonchus contortus. Int. J. Parasitol. 25, 1281-1289.

Piacenza, L., Acosta, D., Basmadjian, I., Dalton, J.P., Carmona, C., 1999. Vaccination with Cathepsin L proteinases and with leucine aminopeptidase induces high levels of protection against fascioliasis in sheep. Infect. Immun. 67, 1954-1961.

Rhoads, M.L., Fetterer, R.H., 1998. Purification and characterisation of a secreted aminopeptidase from adult Ascaris suum. Int. J. Parasitol. 28, $1681-1690$.

Ross, A.G., Bartley, P.B., Sleigh, A.C., Olds, G.R., Li, Y., Williams, G.M., McManus, D.P., 2002. Schistosomiasis. N. Engl. J. Med. 346, $1212-1220$.

Sajid, M., McKerrow, J.H., 2002. Cysteine proteases of parasitic organisms. Mol. Biochem. Parasitol. 120, 1-21.

Sajid, M., McKerrow, J.H., Hansell, E., Mathieu, M.A., Kimberley, D.L., Hsieh, I., Greenbaum, D., Bogyo, M., Salter, J.P., Kim, K.C., Franklin, C., Kim, J.H., Caffrey, C.R., 2003. Functional expression and characterization of Schistosoma mansoni cathepsin B and its transactivation by an endogenous asparaginyl endopeptidase. Mol. Biochem. Parasitol. 131, 65-75.

Strater, N., Lipscomb, W.N., 1998. Clan MF containing co-catalytic leucyl aminopeptidases. In: Barrett, A.J., Rawlings, N., Woessner, J.F. (Eds.), Handbook of Proteolytic Enzymes, Academic Press, London, pp. 1382-1389.

Taylor, A., 1993. Aminopeptidases: towards a mechanism of action. Trends Biochem. Sci. 18, 167-171.

Thompson, J.D., Gibson, T.J., Plewniak, F., Jeanmougin, F., Higgins, D.G., 1997. The CLUSTAL_X interface: flexible strategies for multiple sequence alignment aided by quality analysis tools. Nucleic Acids Res. 15, 4876-4882.

Tort, J., Brindley, P.J., Knox, D., Wolfe, K.H., Dalton, J.P., 1999. Helminth proteinases and their associated genes. Adv. Parasitol. 43, $162-168$.

Verjovski-Almeida, S., DeMarco, R., Martins, E.A.L., Guimaraes, P.E.M., Ojopi, E.P.B., Paquola, A.C.M., Piazza, J.P., Nishiyama, M.J. Jr., Kitajima, J.P., Adamson, R.E., Ashton, P.D., Bonaldo, M.F., Coulson, P.S., Dillon, G.P., Farias, L.P., Gregorio, S.P., Ho, P.L., Leite, R.A., Malaquias, L.C.C., Marques, R.C.P., Miyasata, P.A., Nascimento, A.L.T.O., Ohlweiler, F.P., Reis, E.M., Ribeiro, M.A., Sa, R.G., Stukart, G.C., Soares, M.B., Gargioni, C., Kawano, T., Rodrigues, V., Madeira, A.M.B.N., Wilson, R.A., Menck, C.F.M., Setubal, J.C., Leite, L.C.C., Dias-Neto, E., 2003. Transcriptome analysis of the acoelomate human parasite Schistosoma mansoni. Nat. Genet. 35, 148-157.

WHO, 1999. Report of the WHO informal consultation on schistosomiasis control. World Health Organization, Geneva, WHO/CDS/CPC/SIP/ 99.2.

Xu, Y.Z., Dresden, M.H., 1986. Leucine aminopeptidase and hatching of Schistosoma mansoni eggs. J. Parasitol. 72, 507-511.

Xu, Y.Z., Matsuda, H., Dresden, M.H., 1988. Effect of praziquantel on Schistosoma mansoni eggs: leucine aminopeptidase (LAP) activity and anti-LAP antibodies. Am. J. Trop. Med. Hyg. 39, 46-51.

Xu, Y.Z., Shawar, S.M., Dresden, M.H., 1990. Schistosoma mansoni: purification and characterization of a membrane-associated leucine aminopeptidase. Exp. Parasitol. 70, 124-133. 